\title{
Characterization of Recombinant Bovine Sperm Hyaluronidase and Identification of an Important Asn-X-Ser/Thr Motif for Its Activity
}

\author{
Chaeri Park $^{1 \dagger}$, Young-Hyun Kim ${ }^{2,3 \dagger}$, Sang-Rae Lee ${ }^{2,3 \dagger}$, Soojin Park ${ }^{4}$, Yena Jung ${ }^{1}$, Youngjeon Lee ${ }^{2,3}$, Ji-Su Kim ${ }^{2,3}$, \\ Taekil Eom ${ }^{5}$, Ju-Sung Kim ${ }^{5}$, Dong-Mok Lee ${ }^{6}$, Bong-Suk Song ${ }^{2,3}$, Bo-Woong Sim ${ }^{2}$, Sun-Uk Kim ${ }^{2,3}$, \\ Kyu-Tae Chang ${ }^{2,3 *}$, and Ekyune Kim ${ }^{1 *}$ \\ ${ }^{1}$ College of Pharmacy, Catholic University of Daegu, Gyeongsan 38430, Republic of Korea \\ ${ }^{2}$ National Primate Research Center (NPRC), Korea Research Institute of Bioscience and Biotechnology (KRIBB), Daejeon 34141, Republic of Korea \\ ${ }^{3}$ Department of Functional Genomics, KRIBB School of Bioscience, Korea University of Science and Technology (UST), Daejeon 34113, \\ Republic of Korea \\ ${ }^{4}$ Graduate School of Medicine, Osaka University, Suita, Osaka 5650871, Japan \\ ${ }^{5}$ College of Applied Life Sciences, the Research Institute for Subtropical Agriculture and Biotechnology, Jeju National University, Jeju 63249, \\ Republic of Korea \\ ${ }^{6}$ Daegyeong Regional Division, Technology Convergence REBD Group, Korea Institute of Industrial Technology, Yeongcheon 38822, \\ Republic of Korea
}

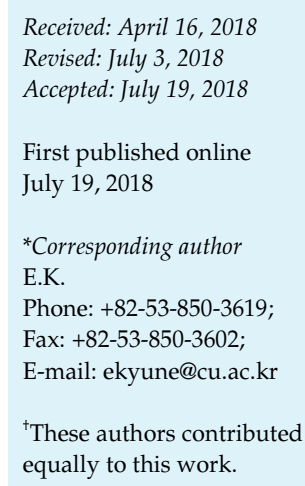

Hyaluronidases are a family of enzymes that catalyse the breakdown of hyaluronic acid, which is abundant in the extracellular matrix and cumulus oocyte complex. To investigate the activity of recombinant bovine sperm hyaluronidase 1 (SPAM1) and determine the effect of the Asn-X-Ser/Thr motif on its activity, the bovine SPAM1 open reading frame was cloned into the mammalian expression vector pCXN2 and then transfected to the HEK293 cell line. Expression of recombinant bovine hyaluronidase was estimated using a hyaluronidase activity assay with gel electrophoresis. Recombinant hyaluronidase could resolve highly polymeric hyaluronic acid and also caused dispersal of the cumulus cell layer. Comparative analysis with respect to enzyme activity was carried out for the glycosylated and deglycosylated bovine sperm hyaluronidase by N-glycosidase F treatment. Finally, mutagenesis analysis revealed that among the five potential N-linked glycosylation sites, only three contributed to significant inhibition of hyaluronic activity. Recombinant bovine SPAM1 has hyaluronan degradation and cumulus oocyte complex dispersion ability, and the N-linked oligosaccharides are important for enzyme activity, providing a foundation for the commercialization of hyaluronidase.

Keywords: Hyaluronidase, hyaluronic acid, cumulus oocyte complex, fertilization

\section{Introduction}

Glycosaminoglycan hyaluronic acid (HA), a polymer consisting of repeating disaccharide units of N-acetyl-Dglucosamine and D-glucuronic acid [1], is a major structural component of the extracellular matrix and the cumulusoocyte complex (COC) [2]. Regulated HA synthesis and degradation are critical for multiple biological processes, including cell migration [3], wound healing [4], malignant transformation [5], tissue turnover [6], fertilization [7], and egg development [8]. Hyaluronidases are responsible for the degradation of HA, and are widely distributed from bacteria to mammals exhibiting endo-beta-N-acetyl hexosaminidases activity, with tetrasaccharides and hexasaccharides as the major end-products along with HA [9]. The three mammalian genes encoding hyaluronidases, 
HYAL1, HYAL2, and HYAL3, are expressed in somatic cells, whereas the HYAL4, HYAL6, and sperm hyaluronidase 1 (SPAM1) genes are expressed in the germ cells and located on human chromosome 3p21.3 and 7q31.3 [10, 11]. Recently, human SPAM1 was produced as a recombinant protein and commercialized; however, its activity is lower than that of the SPAM1 protein found in large animals.

Although controversies remain over its function and safety, SPAM1 is frequently used in cosmetic surgery (https://www.mdedge.com) and for in vitro fertilization [12], and its subcutaneous injection can increase the penetration of vaccines and toxins [13]. At present, commercial testis-specific hyaluronidase is mainly isolated from bulls and sheep, and the protein homology is only $\sim 55 \%$ with human SPAM1. To overcome these limitations toward development of a more effective and biologically functional commercialized SPAM1, we produced and characterized a high-activity bovine recombinant hyaluronidase using a mammalian expression vector.

\section{Materials and Methods}

\section{Tissue Sample Collection}

A fresh bull testis was purchased from a local slaughterhouse (Korea), immediately flushed with ice-cold buffer (20 mM Tris$\mathrm{HCl}, \mathrm{pH} 7.4,150 \mathrm{mM} \mathrm{NaCl}$ ), and then placed in liquid nitrogen for deep freezing [12].

\section{Total RNA Extraction and cDNA Cloning of Bovine SPAM1 (bSPAM1)}

Total cellular RNA was extracted from the bull testis using ISOGEN (Nippon Gene, Japan) and cDNA was synthesized by oligo (dT) priming using the SuperScript III First-Strand Synthesis System (Invitrogen, USA) [2]. To amplify the cDNA fragments encoding bull SPAM1, two oligonucleotide primers were designed based on a bovine expressed sequence tag (GenBank Accession No. NM_001008413.3). The oligonucleotide sequences for the primers were as follows: 5'-CTCGAGGCCACCATGAGAATG CTGAGGCGCCACCA-3' (sense) and 5'-CTCGAGTTAATAGGT TGTTTGATTTTTAAT-3' (antisense).

Polymerase chain reaction (PCR) was performed for 35 cycles at $94^{\circ} \mathrm{C}$ for $60 \mathrm{sec}, 60^{\circ} \mathrm{C}$ for $60 \mathrm{sec}$, and $72^{\circ} \mathrm{C}$ for $90 \mathrm{sec}$ with a PCR thermal cycler (Biometra, Germany). The reverse transcriptionPCR products corresponding to the $b S P A M 1$ gene were cloned and sequenced. Following electrophoresis on a $1 \%$ agarose gel, the desired PCR band was excised with a razor blade. The gel fragment was purified using a gel extraction kit (Elpis Biothech, Korea) in accordance with the manufacturer's guidelines. The purified DNA fragment was then cloned in Escherichia coli DH5 $\alpha$ cells using the pEGM-T easy kit (Promega, USA). After isolation of plasmid DNA, inserts were sequenced with the vector-specific T7 and SP6 sequencing primers (Promega, USA) on an ABI 3100 DNA sequencer (Applied Biosystems, USA).

\section{Preparation of Protein Extracts and Sodium Dodecyl Sulfate-} Polyacrylamide Gel Electrophoresis (SDS-PAGE)

Proteins were extracted by mincing and grinding the bovine testis at $100 \times g$ on ice for $2 \mathrm{~h}$ in a buffer consisting of $20 \mathrm{mM}$ Tris$\mathrm{HCl}, \mathrm{pH} 7.4,150 \mathrm{mM} \mathrm{NaCl}$. The sample was then centrifuged at $10,000 \times g$ for $10 \mathrm{~min}$ at $4^{\circ} \mathrm{C}$, and then the proteins retained in the supernatant were analyzed. Denatured proteins were separated by SDS-PAGE.

\section{Zymography by Agarose Gel Electrophoresis}

Fifty microliters of $1 \%$ hyaluronan (Sigma-Aldrich) was digested with proteins $(1 \mu \mathrm{g})$ extracted from transfected HEK293 cells for $12 \mathrm{~h}$, and the digested hyaluronan was separated on $0.8 \%$ agarose gels. For visualization and analysis, the agarose gels were stained with $0.5 \%$ Alcian Blue $8 \mathrm{GX}$.

\section{Mutagenesis of bSPAM1}

The plasmid pCXN2-bSPAM1 gene fragment was used as a mutagenesis template. All primers designed to introduce the sitedirected mutation were synthesized by COSMOgenetech (Fig. 4A and Table 1). PCR amplifications were carried out with the Expand TM High Fidelity PCR system [14]. The 50- $\mu$ l PCR reaction was carried out with $100 \mathrm{ng}$ templates, $10 \mathrm{mM}$ primer pair, $200 \mathrm{mM}$ dNTPs, and $2 \mathrm{U}$ of DNA polymerase. The extension reaction was initiated by pre-heating the reaction mixture to $95^{\circ} \mathrm{C}$ for $5 \mathrm{~min}$, followed by 20 cycles of $95^{\circ} \mathrm{C}$ for $1 \mathrm{~min}$ and $55^{\circ} \mathrm{C}$ for $1 \mathrm{~min}$, and final extension at $72^{\circ} \mathrm{C}$ for $14 \mathrm{~min}$. After the PCRamplification product was digested by $\mathrm{DpnI}$ at $37^{\circ} \mathrm{C}$ for $3 \mathrm{~h}$ to destroy the plasmid fragments, it was purified with a PCR purification kit. A $10-\mu l$ aliquot of the purified PCR product was

Table 1. Number of primer sequences for mutagenesis.

\begin{tabular}{lll}
\hline & \multicolumn{1}{c}{ Sense primer $\left(5^{\prime}\right.$ to $\left.3^{\prime}\right)$} & \multicolumn{1}{c}{ Anti sense primer $\left(5^{\prime}\right.$ to $\left.3^{\prime}\right)$} \\
\hline SPAM_MT_No.1 & CCTATTACATACCAggtaCCAGCGTGGGCTTGGC & GCCAAGCCCACGCTggtaCCTGGTATGTAATAGG \\
SPAM_MT_No.2 & ATGTGGGGCAGTCTCggtaCCAGCTTATCTATGC & GCATAGATAAGCTggtaCCGAGACTGCCCCACAT \\
SPAM_MT_No.3 & TAGGCACTTACTTGggtaCCACACTGAATCCTTA & TAAGGATTCAGTGTggtaCCCAAGTAAGTGCCTA \\
SPAM_MT_No.4 & ATCCTTACATAATCggtaCCACCCTAGCCGCCAA & TTGGCGGCTAGGGTggtaCCGATTATGTAAGGAT \\
SPAM_MT_No.5 & TACAAGGAAACACTGGggtaCCAGCGACTATCTTCACC & GGTGAAGATAGTCGCTggtaCCCCAGTGTTTCCTTGTA \\
\hline
\end{tabular}


A

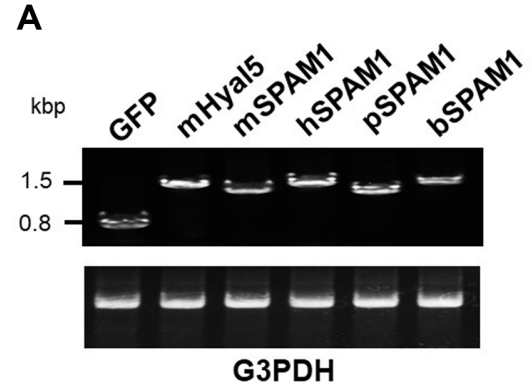

B

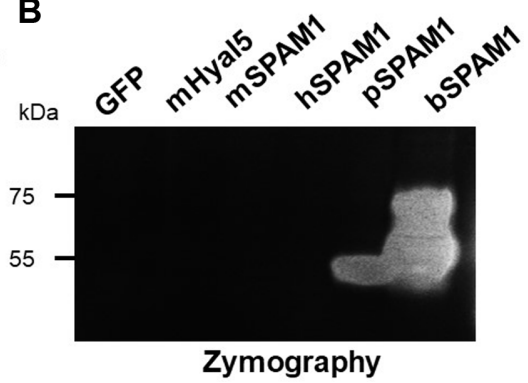

Fig. 1. Expression of various mammalian SPAM1 genes in HEK293 cells.

(A) Detection of transcripts for various SPAM1 genes by RT-PCR with mRNA isolated from HEK293 cells. G3PDH was used as an internal standard. (B) Activity of SPAM1 hyaluronidase in HEK293 cells. Proteins in Triton X-100 extracts from SPAM1-transformed HEK293 cells were separated by SDS-PAGE under reducing conditions and subjected to a zymography assay. GFP; green fluorescent protein, mHyal5; mouse hyaluronidase 5, mSPAM1; mouse SPAM1, hSPAM1; human SPAM1, pSPAM1; pig SPAM1, bSPAM1; bovine SPAM1, G3PDH; Glyceraldehyde 3phosphate dehydrogenase.

then transformed into XL1-Blue chemocompetent cells and inoculated on Luria-Bertani (LB) plates containing $100 \mathrm{mg} / \mathrm{ml}$ ampicillin. A total of three colonies were selected, and their plasmids were isolated by miniprep and subjected to DNA sequencing.

\section{Dispersal of Cumulus Cells}

Eggs that were tightly packed with a COC mass were collected from the oviductal ampulla of superovulated ICR mice 14-15 h after human chorionic gonadotropin injection, and then placed in a $0.1 \mathrm{ml}$ drop of a modified Krebs-Ringer bicarbonate solution (TYH medium) covered with mineral oil. The bSPAM1-transformed HEK293 cell extracts ( $3 \mathrm{mg}$ of protein $/ \mathrm{ml}$ ) were mixed with the $\mathrm{COC}$ in TYH medium and incubated at $37^{\circ} \mathrm{C}$ for 30 min under $5 \%$ $\mathrm{CO}_{2}$ in air. The dispersal of cumulus cells was observed on an IX71 microscope (Olympus, Japan) equipped with a DP-12 camera, as described previously [16].

\section{Results and Discussion}

To determine the hyaluronidase activity of bovine Sperm adhesion molecule 1 (bSPAM1), the bSPAM1 gene was cloned into the pCXN2 expression vector for overexpression in HEK293 cells. In silico analysis using gene information from the National Center of Biotechnology Information showed that the DNA sequence of bSPAM1 is synthesized as a single-chain protein comprising 407 amino acid residues with a calculated molecular mass of $44,013 \mathrm{Da}$. As compared to mouse SPAM1 (mSPAM1), bSPAM1 has an approximately 20-residue additional sequence at the cytoplasmic tail domain. On the basis of homology with the mouse sequences, bSPAM1 was predicted to contain multidomains, including a signal peptide, hyaluronidase domain, ZP-binding domain, and a glycophosphatidylinositol (GPI) anchor domain. Unlike mSPAM1, which consists of double chains responsible for the endoproteolytic cleavage of the Arg347-Ala 348 site through covalently linked disulfide bridges [10], the mature bovine SPAM1 (bSPAM1) protein exists as a single protein molecule and does not contain an Arg-Ala site. The sequences of the hyaluronidase and ZP-binding domains showed $81.3 \%$ and $79.7 \%$ similarity, respectively, between mouse and bovine SPAM1, while the sequence of the GPI anchor domain showed relatively lower similarity at $45.3 \%$. The overall homology between mouse and bovine SPAM1 was estimated at $68 \%$.

To determine whether the bSPAM1 gene possesses hyaluronan degradation activity, we initially cloned variants of SPAM1 from mouse, human, porcine, cat and bovine testis cDNA libraries into the pCXN2 vector (Fig. 1A). Comparison of the activities of recombinant hyaluronidases from cattle, pig, goat, cat, rabbit, and mouse SPAM1 genes, showed that recombinant bSPAM1 showed the strongest activity. In contrast, almost no activity was observed by recombinant SPAM1 from the other animals except for the

Table 2. SPAM1 amino acid sequence homology among the mammals.

\begin{tabular}{ccccc}
\hline $\begin{array}{c}\text { SPAM1 molecule } \\
\text { (Accession No.) }\end{array}$ & $\begin{array}{c}\text { Pig } \\
(\text { NM_001174044) }\end{array}$ & $\begin{array}{c}\text { Human } \\
(\text { NM_001174044) }\end{array}$ & $\begin{array}{c}\text { Mouse } \\
\text { (NM_001079875) }\end{array}$ & $\begin{array}{c}\text { Cat } \\
(\text { XM_011280528) }\end{array}$ \\
\hline $\begin{array}{c}\text { Bovine (\%) } \\
\text { (NM_001008413) }\end{array}$ & 69.27 & 63.55 & 56.30 & 64.79 \\
\hline
\end{tabular}


0

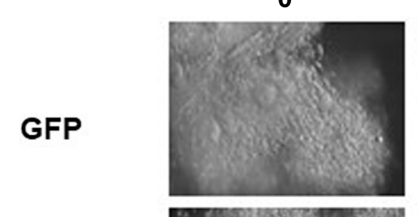

Mouse

SPAM1

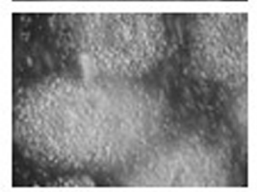

Mouse

HYAL5
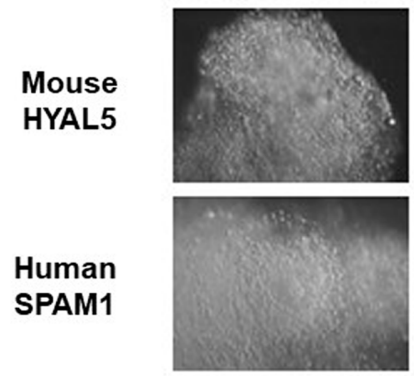

Pig

SPAM1
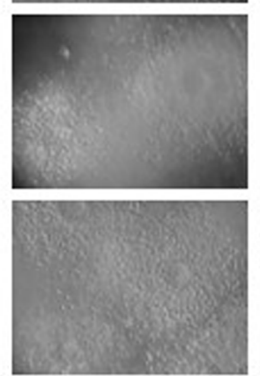

SPAM1

NT

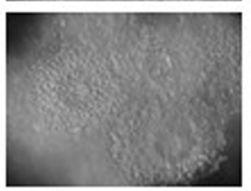

Hylase

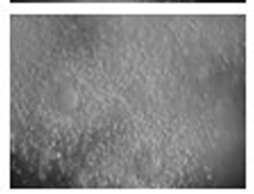

15
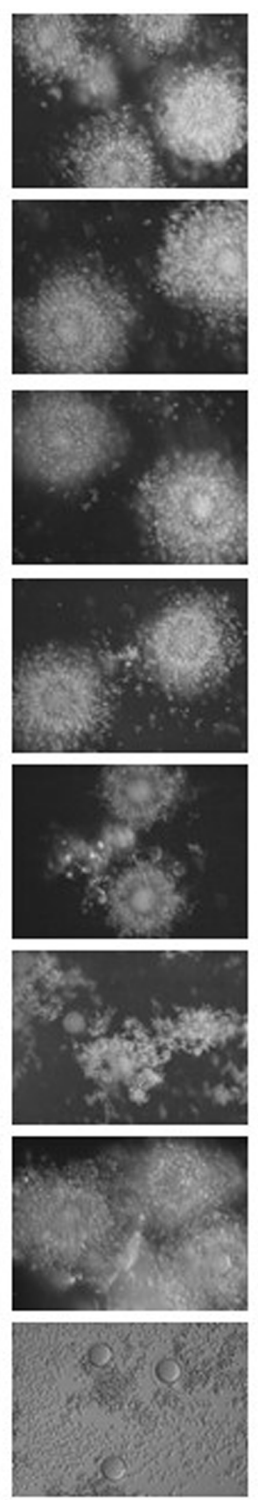

30
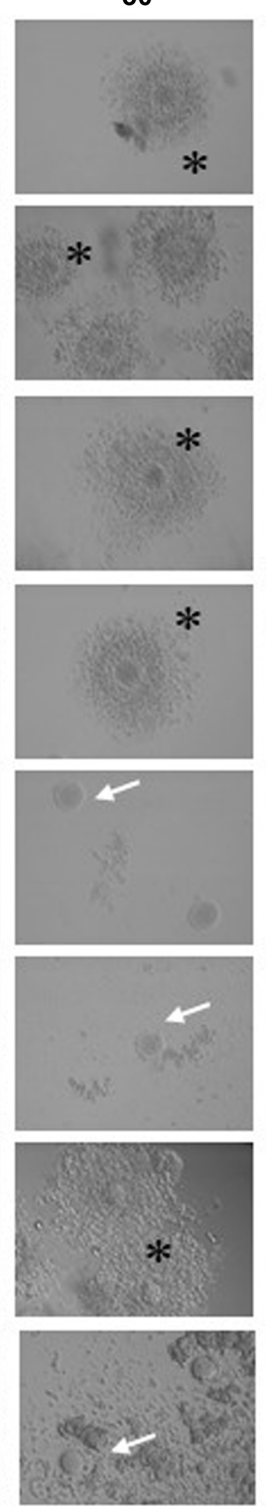

60
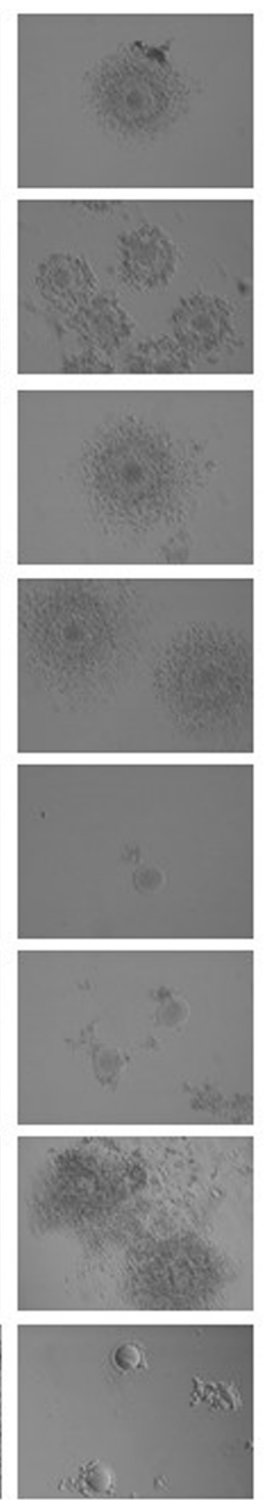

Fig. 2. Dispersal of COC mass.

COCs were incubated for $0,15,30$, and 60 min with the extracts. Asterisks indicate undispersed COC, while the arrowheads indicate oocyte after COC dispersal.

pig (Fig. 1B). This is likely due to the high homology between pigs and bulls (Table 2). In addition, we examined whether the recombinant bSPAM1 could degrade the COC, which is the first step in correcting the major role of sperm hyaluronidase. As shown in Fig. 2, bovine and pig derived hyaluronidase had COC dispersing ability, whereas mouse and human recombinant hyaluronidase did not show hyaluronidase activity. Sperm hyaluronidase is known to be attached to the membrane surface by the GPI anchor, implying that the protein activity could be influenced by
N-linked glycosylation. N-linked glycosylation, which is generally known to be present in eukaryotes and a wide range of archaea, involves glycosylation of an asparagine (Asn) residue of the tripeptide sequence Asn-X-Ser or Asn-X-Thr, called the sequon sequence, by a transferase oligosaccharide [17]. This sugar chain is known to play an important role in the stabilization of glycoproteins, their expression on the cell surface, and intercellular interactions [18], although the exact function has not yet been elucidated. To further confirm the possible function of N-linked 
A

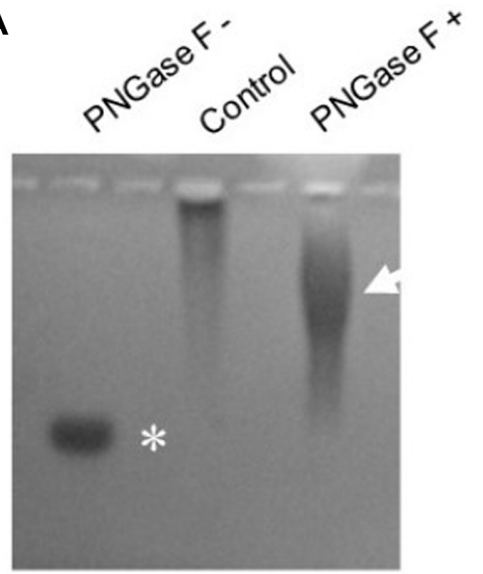

B

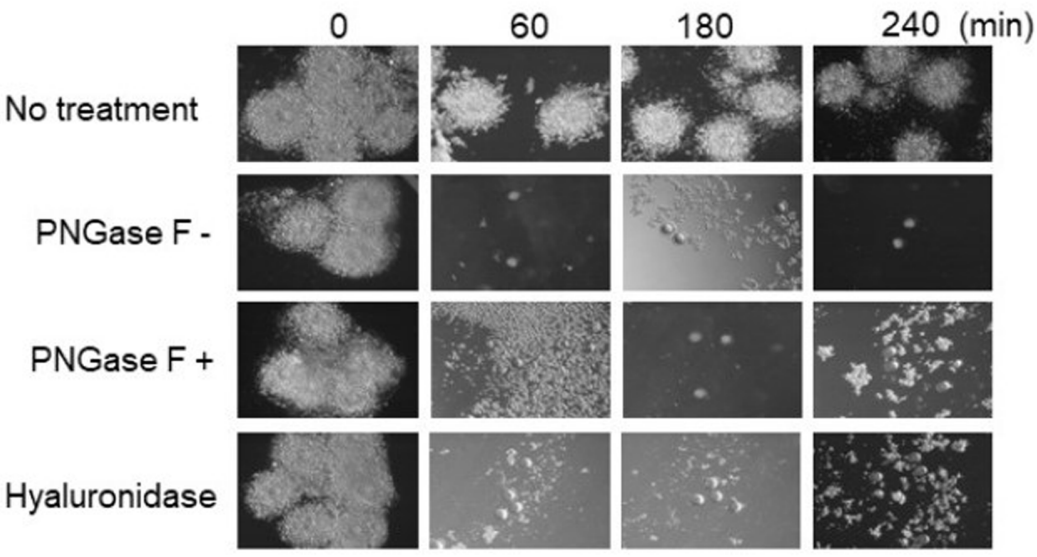

Fig. 3. Hyaluronic acid degradation assay with peptide-N-glycosidase F (PNGase F).

(A) Protein from each sample $(50 \mu \mathrm{g})$ was run on a $0.8 \%$ agarose gel followed by in-gel hyaluronic acid (HA) detection using Alcian blue staining. Stained HA was visualized by illumination. An asterisk indicates cleaved HA fragment. The arrowhead indicates non-cleaved HA polymer. Lane 1, PNGase F untreated sample; lane 2, HA sample; lane 3, PNGase F treated sample. (B) Dispersal of cumulus cells with glycosidase-digested hyaluronidase.

glycosylation from bovine sperm extracts, we next carried out an N-glycosidase F (PNGase F) digestion assay [19]. The hyaluronidase activity was confirmed in the control fraction without PNGase F, but not in the PNGase F-reacted fraction (Fig. 3). Moreover, the Asn residue of each of the five hypothetical N-linked glycosylation sites in bSPAM1 were substituted with a Gly residue to evaluate the effect of the mutation on the ability of the enzyme to degrade HA

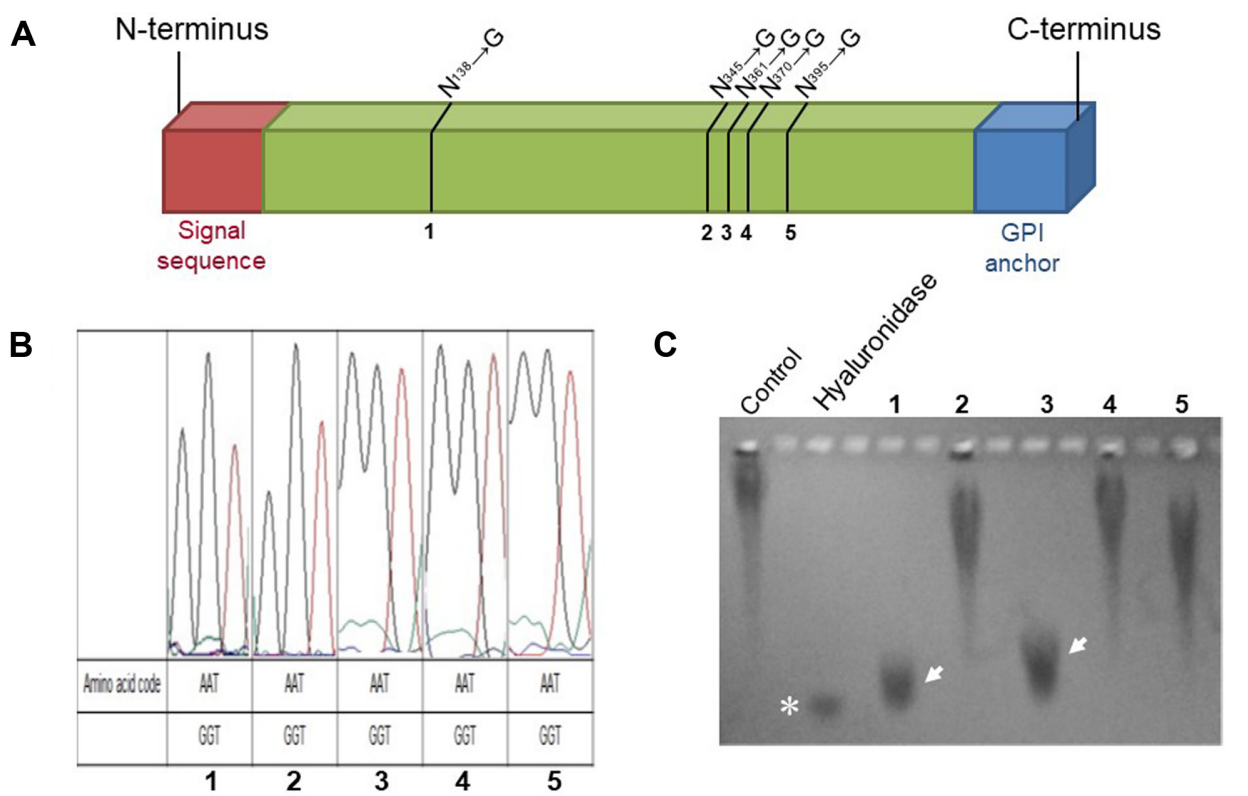

Fig. 4. Mutagenesis of Asn-X-Ser/Thr sites in recombinant bSPAM1.

(A) Schematic representation of mutant SPAM1. Five asparagine sites were replaced by glycine. (B) Sequence analysis. The mutants in which Asn of the Asn-X-Ser/Thr site was replaced were evaluated by sequencing. (C) Wild type bSPAM1 and mutant protein with Gly substituted for Asn were produced from HEK293 cells. After incubation between mutants and 1\% high polymer hyaluronic acid in 1× PBS, the samples were separated by $0.8 \%$ agarose gel electrophoresis and stained with Alcian blue. 


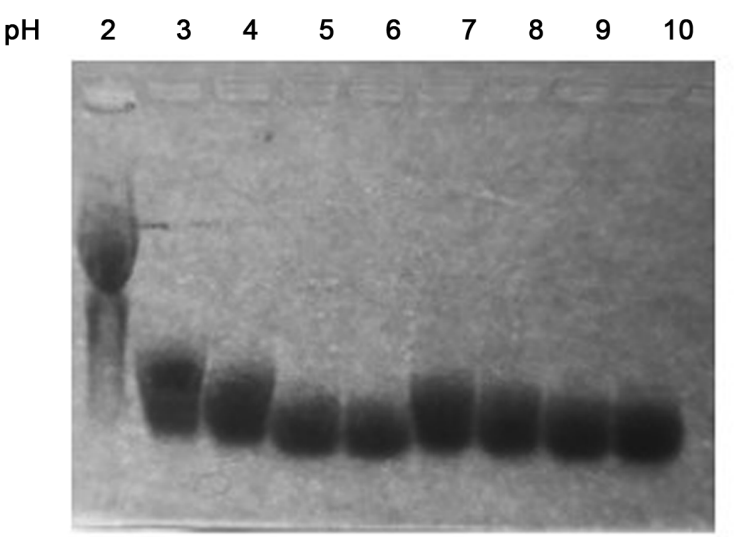

Fig. 5. Hyaluronic acid degradation inhibition assay with varying $\mathrm{pH}$. Five micrograms of soluble proteins per lane from bSPAM1-expressing HEK293 cells was reacted with molecules of hyaluronic acid and separated by $0.8 \%$ agarose gel electrophoresis followed by staining with Alcian blue.

(Figs. 4A and 4B). As shown in Fig. 4C, among the five Asn residues that were mutated, the $\mathrm{Asn}^{395}$ mutation was found to be most important for hyaluronan degradation. In addition, the $\mathrm{Asn}^{345}$ and $\mathrm{Asn}^{375}$ mutations appeared to play a role in the degradation of HA, whereas $\mathrm{Asn}^{138}$ and $\mathrm{Asn}^{361}$ did not have a significant effect on HA decomposition. $\mathrm{Asn}^{395}$ is present in most mammalian SPAM1 proteins, whereas $\mathrm{Asn}^{138}$ is only found in bovine SPAM1.

The optimal $\mathrm{pH}$ for mSPAM1 hyaluronidase activity was reported to be in the range of 5-7 [10]. This corresponds to the estimated intra-acrosomal $\mathrm{pH}$ values of mouse sperm of 5.3 and 6.2 immediately after sperm preparation and $2 \mathrm{~h}$ after capacitation, respectively, in TYH medium [20]. To determine the optimal $\mathrm{pH}$ of the recombinant bSPAM1 (rbSPAM1) molecule, we evaluated its HA activity $\mathrm{pH}$ conditions from 2-9. As shown in Fig. 5, surprisingly, rbSPAM1 activity was detected at all $\mathrm{pH}$ values tested except for $\mathrm{pH} 2$.

In summary, rbSPAM1 shows high HA degradation activity, and we further confirmed that the Asn-X-Ser/Thr sequence, which is conserved in most mammals, is essential for this activity. If the stability of this recombinant protein can be guaranteed with further validation, it will have great potential for commercial development given its ability to overcome current limitations in commercially available hyaluronidases.

\section{Acknowledgments}

This research was supported by National Research
Foundation of Korea Grant, which is funded by the Korean Government (NRF-2017R1D1A1B03031420) and the Korea Research Institute of Bioscience and Biotechnology (KRIBB) Research Initiative Program Grants (KGM4241844 \& KGM4251824).

\section{Conflict of Interest}

The authors have no financial conflicts of interest to declare.

\section{References}

1. Zhu Y, Kruglikov IL, Akgul Y, Scherer PE. 2018. Hyaluronan in adipogenesis, adipose tissue physiology and systemic metabolism. Matrix Biol.

2. Kim E, Kim JS, Lee Y, Song BS, Sim BW, Kim SU, et al. 2013. Molecular cloning, characterization of porcine IZUMO1, an IgSF family member. Reprod. Domest Anim. 48: 90-97.

3. Viola M, Karousou E, D’Angelo ML, Caon I, De Luca G, Passi A, et al. 2015. Regulated hyaluronan synthesis by vascular cells. Int. J. Cell Biol. 2015: 208303.

4. Aya KL, Stern R. 2014. Hyaluronan in wound healing: rediscovering a major player. Wound Repair Regen. 22: 579-593.

5. Nguyen N, Kumar A, Chacko S, Ouellette RJ, Ghosh A. 2017. Human hyaluronic acid synthase-1 promotes malignant transformation via epithelial-to-mesenchymal transition, micronucleation and centrosome abnormalities. Cell Commun. Signal. 15: 48.

6. Stern R, Kogan G, Jedrzejas MJ, Soltes L. 2007. The many ways to cleave hyaluronan. Biotechnol. Adv. 25: 537-557.

7. Nishihara T, Morimoto Y. 2017. Evaluation of transfer media containing different concentrations of hyaluronan for human in vitro fertilization. Reprod. Med. Biol. 16: 349-353.

8. Marei WF, Ghafari F, Fouladi-Nashta AA. 2012. Role of hyaluronic acid in maturation and further early embryo development of bovine oocytes. Theriogenology 78: 670-677.

9. Stern R, Jedrzejas MJ. 2006. Hyaluronidases: their genomics, structures, and mechanisms of action. Chem. Rev. 106: 818-839.

10. Kim E, Baba D, Kimura M, Yamashita M, Kashiwabara S, Baba T. 2005. Identification of a hyaluronidase, Hyal5, involved in penetration of mouse sperm through cumulus mass. Proc. Natl. Acad. Sci. USA 102: 18028-18033.

11. Kimura M, Ishida K, Kashiwabara S, Baba T. 2009. Characterization of two cytoplasmic poly(A)-binding proteins, PABPC1 and PABPC2, in mouse spermatogenic cells. Biol. Reprod. 80: 545-554.

12. Yoon S, Chang KT, Cho H, Moon J, Kim JS, Min SH, et al. 2014. Characterization of pig sperm hyaluronidase and improvement of the digestibility of cumulus cell mass by recombinant pSPAM1 hyaluronidase in an in vitro fertilization assay. Anim. Reprod. Sci. 150: 107-114. 
13. Mirjamali NA, Soufian S, Molaee N, Abbasian SS, Abtahi H. 2014. Cloning and expression of the enzymatic region of Streptococcal hyaluronidase. Iran J. Basic Med. Sci. 17: 667-672.

14. Shin SH, Shin GW, Yim SH, Jung SH, Jung GY, Chung YJ. 2011. Strategy for high-fidelity multiplex DNA copy number assay system using capillary electrophoresis devices. Electrophoresis 32: 1837-1843.

15. Kim Y, Cheon S, Min CK, Sohn KM, Kang YJ, Cha YJ, et al. 2016. Spread of mutant middle east respiratory syndrome coronavirus with reduced affinity to human CD26 during the South Korean outbreak. MBio 7: e00019.

16. Kang W, Zhou C, Koga Y, Baba T. 2010. Hyaluronandegrading activity of mouse sperm hyaluronidase is not required for fertilization. J. Reprod. Dev. 56: 140-144.

17. Geetha-Habib M, Park HR, Lennarz WJ. 1990. In vivo Nglycosylation and fate of Asn-X-Ser/Thr tripeptides. J. Biol. Chem. 265: 13655-13660.

18. Gabius HJ. 2018. The sugar code: Why glycans are so important. Biosystems 164: 102-111.

19. Tarentino AL, Gomez CM, Plummer TH Jr. 1985. Deglycosylation of asparagine-linked glycans by peptide: $\mathrm{N}$ glycosidase F. Biochemistry 24: 4665-4671.

20. Liu L, Nutter LM, Law N, McKerlie C. 2009. Sperm freezing and in vitro fertilization in three substrains of C57BL/6 mice. J. Am. Assoc. Lab. Anim. Sci. 48: 39-43. 